\title{
Measuring anxiety \& depression in the post critical care population - comparison of the hospital anxiety and depression scale and intensive care psychological assessment tool
}

\author{
N Mason*, D Egan, S O'Loghlen, D Conway \\ From ESICM LIVES 2015 \\ Berlin, Germany. 3-7 October 2015
}

\section{Introduction}

The impact of critical illness on psychological wellbeing can be severe with patients reporting high levels of anxiety \& depression [1]. The psychological impact of critical illness can be long lasting, affecting both patients and their families and impacting on quality of life following hospital discharge [2]. A minority of patients will develop posttraumatic stress disorder following the acute illness and the NICE guideline CG83 recommends that patients are assessed for acute distress and risk of future psychological morbidity [3].

The Hospital Anxiety and Depression Scale was developed as a snapshot assessment of psychological morbidity for hospital in-patients and has been validated in Critical Care Follow Up Clinic patients with a cut-off of $\geq 8 / 14$ for each domain for mild distress and $\geq 11 / 14$ for significant morbidity [4].

The IPAT tool has been validated in critically ill patients as a screening tool to detect acute distress in critical care patients once they are awake, alert \& orientated, with a cut off $\geq 7$ / 20 indicating morbidity [5]. It has not been previously reported immediately following Critical Care discharge.

\section{Objectives}

The aims of this survey are to measure and compare screening of acute distress in patients recovering from critical illness recently transferred to the general ward, using two different assessment tools - HADS and IPAT

Manchester Royal Infirmary, Central Manchester University Hospitals NHS Foundation Trust, Manchester Academic Health Science Centre, Manchester, United Kingdom

(c) 2015 Mason et al.; This is an Open Access article distributed under the terms of the Creative Commons Attribution License (http:// creativecommons.org/licenses/by/4.0), which permits unrestricted use, distribution, and reproduction in any medium, provided the original work is properly cited.

\begin{abstract}
Methods
Prospective data was collected from patients within 48 hours of discharge from the critical care unit in patients who received any level 3 critical care or level 2 critical care for greater than 5 days. The assessment tools were administered by a trained nurse to all patients who were awake and could comprehend the questions. Data were collected between December 2014 - January 2015 and uploaded to an electronic database as part of routine care in accordance with NICE CG83 and analysed with Stats Direct.
\end{abstract}

\section{Results}

68 patients completed HADS \& IPAT simultaneously. 7 patients scored $\geq 8 \& 5 \geq 11$ for HAD Anxiety. 12 scored $\geq 8 \& 7 \geq 11$ for HAD depression. 10 scored $\geq 11$ on the IPAT. Correlation of Total HADS and IPAT scores $r=$ 0.710526 . The median time taken to administer HADS was 4 minutes and IPAT 2 minutes. 3 patients scored $\geq 8$ HAD anxiety yet did not score $\geq 7$ on IPAT.

\section{Conclusions}

IPAT can be administered to most patients following critical care discharge. There was reasonable correlation with HADS. IPAT scored low in patients with no recollection of ICU even when HAD anxiety score was elevated. This merits further investigation.

Published: 1 October 2015

doi:10.1186/2197-425X-3-S1-A963

Cite this article as: Mason et al:: Measuring anxiety \& depression in the post critical care population - comparison of the hospital anxiety and depression scale and intensive care psychological assessment tool. Intensive Care Medicine Experimental 2015 3(Suppl 1):A963. 